Document downloaded from:

http://hdl.handle.net/10251/85244

This paper must be cited as:

Galve-Higón, JM.; Gasulla Mestre, I.; Sales Maicas, S.; Capmany Francoy, J. (2016).

Reconfigurable Radio Access Networks Using Multicore Fibers. IEEE Journal of Quantum Electronics. 52(1):1-7. doi:10.1109/JQE.2015.2497244.

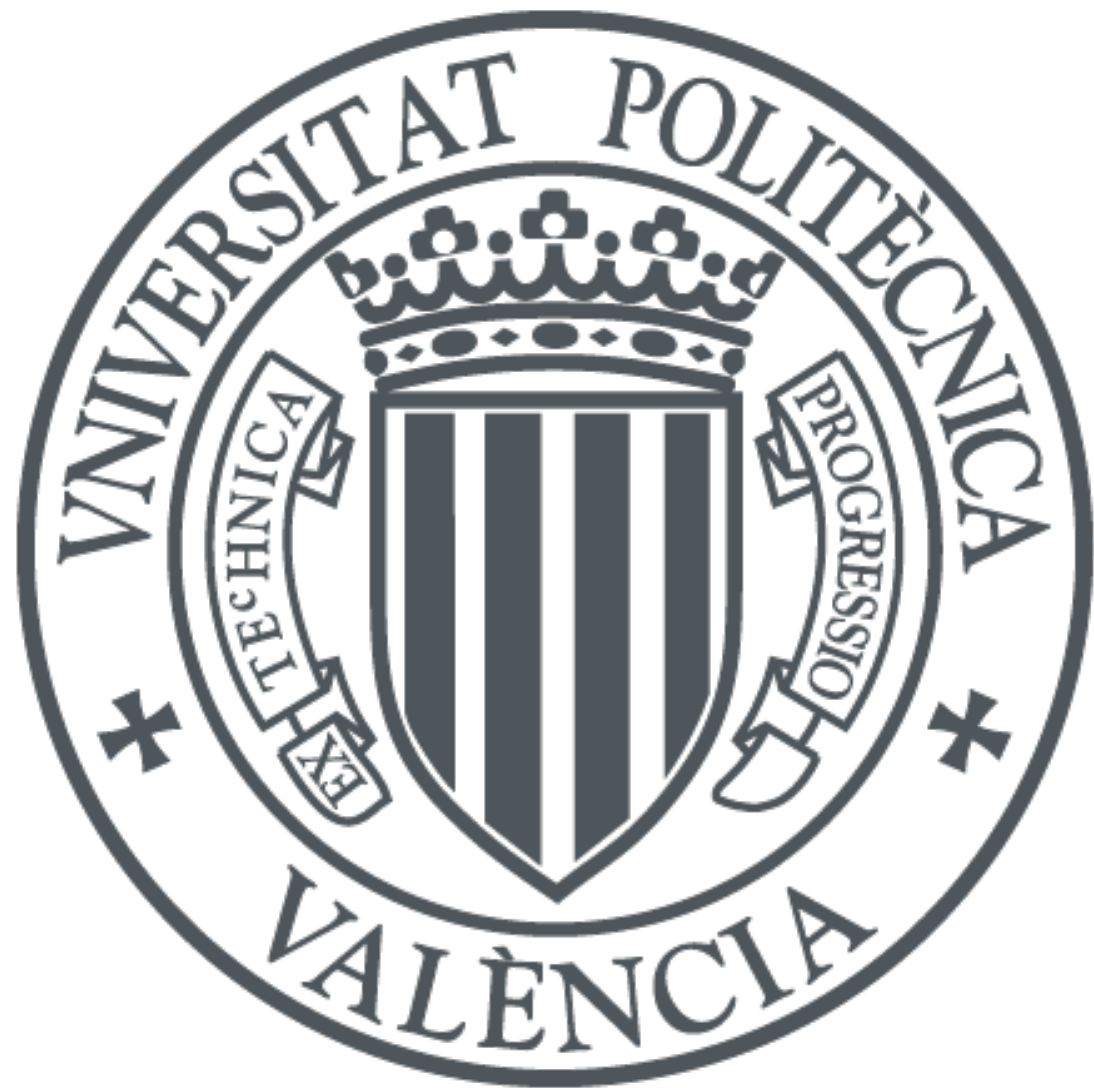

The final publication is available at

http://doi.org/10.1109/JQE.2015.2497244

Copyright Institute of Electrical and Electronics Engineers (IEEE)

Additional Information

"(c) 2016 IEEE. Personal use of this material is permitted. Permission from IEEE must be obtained for all other users, including reprinting/ republishing this material for advertising or promotional purposes, creating new collective works for resale or redistribution to servers or lists, or reuse of any copyrighted components of this work in other works." 


\title{
Reconfigurable Radio Access Networks using Multicore Fibers
}

\author{
José Manuel Galve, Ivana Gasulla, Salvador Sales, Senior Member, IEEE, José Capmany, Fellow, IEEE
}

(Invited Paper)

\begin{abstract}
We propose the use of spatial division multiplexing supported by multicore fibers (MCFS) to implement a new generation of flexible and capacity reconfigurable C-RAN fronthaul architectures capable of addressing their main present and future challenges. We show that for the majority of radio access scenarios where front-haul optical links are less than 10-km long, the impact of inter-core crosstalk on the electrical carrier-tonoise ratio can be neglected and, thus, link design can be carried independently core by core. In addition, this MCF-based approach, which is compatible with SDN and NFV, can also support the integration of a passive optical network overlay.
\end{abstract}

Index Terms - Microwave Photonics, Radio Access networks, radio over fiber systems, multicore fibers, spatial division multiplexing.

\section{INTRODUCTION}

$\mathrm{C}$ ENTRALISED or Cloud Radio Access Networks (CRANs) have been proposed for $5 \mathrm{G}$ converged fiberwireless access networks [1-4]. Based first on the physical separation of the baseband processing unit (BBU) and the Remote Radio Head (RRH) of a traditional Base Station (BS) and, secondly, on the principle of hosting and pooling the baseband processing resources corresponding to different remote BSs in a shared Central Office (CO), the C-RAN concept brings considerable advantages. Amongst them, it is worth mentioning operational and capital expenditure savings, reduced power consumption and improved radio performance derived from the potential low latency between BSs, enabling the possibility of implementing Coordinated Multi-Pont (CoMP) and Long Term Evolution Advanced (LTE-A) systems. C-RANs require the introduction of an optical fronthaul segment for the remote feeding from the $\mathrm{CO}$ of different BS RRHs and antennas, which may in addition feature multiple input, multiple output (MIMO) radiating elements per sector. Digital Radio-over-Fiber (DRoF) is employed to transport the baseband signal from a given BBU to its corresponding $\mathrm{RRH}$, and vice versa, using one of the

Manuscript received September 15, 2015. This work was supported in part by GVA PROMETEO 2013/012, TEC2013-50552-EXP and TEC201460378-C2-1-R projects. Ivana Gasulla thanks the support of Spanish Ministry of Economy and Commerce through the Ramón y Cajal Program.

The authors are with the Institute of Telecommunications and Multimedia (ITEAM), Universitat Politècnica de València, Edificio 8G, Camino de Vera s/n, 46021 Valencia, Spain. (e-mail: jcapmany@iteam.upv.es). protocols defined in the Common Public Radio Interface (CPRI) or the Open Base Station Architecture Initiative (OBSAI).

CRANs face a number of important challenges. The first one is related to bit rate requirements. For instance, CPRI requires very high symmetric bit rates compared to real data rate on the user equipment. For instance, the transport of 5 contiguous $20-\mathrm{MHz}$ LTE-A channels requires a bit rate of $6.144 \mathrm{~Gb} / \mathrm{s} /$ sector. This figure can grow up to almost 50 $\mathrm{Gb} / \mathrm{s} /$ sector if the capacity is increased by means of exploiting, for instance, 8x8 MIMO. To solve this limitation some contributions have suggested the direct use of analog Radio over Fiber (RoF) between the BBU and the RRHs [5], [6]. The second challenge is related to fiber availability. In principle, at least 6 fibers are required per 3-sector cell for single antenna operation. If MIMO configurations are targeted, then some kind of multiplexing (such as wavelength division multiplexing (WDM)) must be considered to cope with the constraint of not increasing the fiber count. A third challenge is the need to support other features, such as capacity increase by carrier aggregation, dynamic capacity allocation as well as centralized control, supervision and management. A final challenge resides in its integration in current and/or evolved versions of passive optical access networks (PONs).

In this paper we propose the use of homogeneous multicore fibers (MCFs) to support CRAN architectures capable of meeting the former challenges though the exploitation of spatial division multiplexing (SDM). MCFs are usually available in 7- and 19-core formats [7] and feature negligible crosstalk values $(<-50 \mathrm{~dB})$ for links below $10 \mathrm{~km}$, which are currently the $96 \%$ of the access networks. We show that MCF-CRAN architectures can be flexible enough to support both DRoF and RoF approaches, capacity upgrade by carrier aggregation and MIMO, CoMP operation and true cloud operation. The key advantage brought by this configuration is the fact that, due to the spatial diversity inherent to MCFs, these features can be enabled by means of electronic spatial switching at the $\mathrm{CO}$, thus allowing software defined networking (SDN) and Network function virtualization (NFV). Furthermore, this configuration is compatible and potentially upgradable using WDM and can support as well a PON overlay. 
The paper is structured as follows: Section II first presents the analog homogeneous MCF link between the CO and one sector of the BS that is the building block of the proposed architecture. It develops the basic link design equations, which must take into account the effect of inter-core crosstalk. It is shown that for most of the practical situations where MCF CO-BS sector links are less than $10-\mathrm{km}$ long the effect of inter-core crosstalk on the electrical carrier to noise ratio (CNR) can be neglected and, therefore, each analog link from the $\mathrm{CO}$ to the $\mathrm{BS}$ and viceversa can be independently designed. Section III investigates the possibility of exploiting the versatility provided by the inherent parallelism of MCF transmission to implement flexible and capacity reconfigurable RAN architectures. Two cases of practical interest are considered, corresponding to pure RoF and DRoF respectively. In each scenario, centralized techniques for capacity reconfiguration are discussed. Section IV considers the potential integration of PONs in the proposed architectures. Finally Section V develops the summary, conclusions and future directions of research.

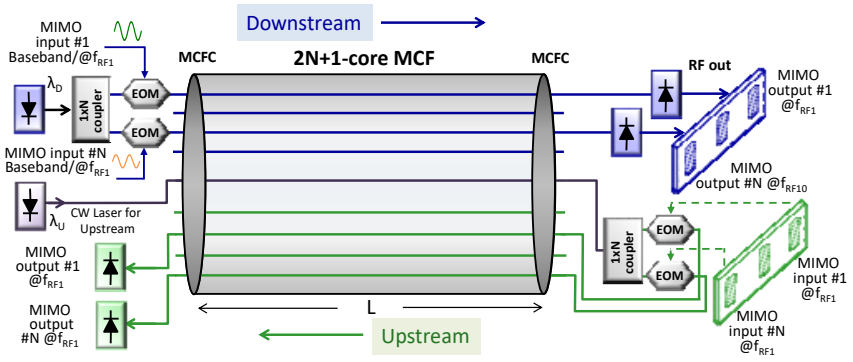

Fig. 1. Link between $\mathrm{CO}$ and $\mathrm{BS}$ sector using a $(2 \mathrm{~N}+1)$-core $\mathrm{MCF}$.

\section{CO TO BS SECTOR SDM OPTICAL LINK}

\section{A. Basic Building Block}

The basic building block of the proposed architecture is a link between the $\mathrm{CO}$ and a single $\mathrm{N}$-antenna sector based on a $(2 N+1)$-core homogeneous MCF, which is shown in Fig. 1. In this work we only consider homogeneous MCFs since heterogeneous MCFs, which provide a different propagation characteristic per node preventing the time matched parallel transmission required for MIMO. Another possibility for SDM is the use of few mode fibers (FMFs), however this scheme brings in our opinion no relevant advantage since it would require complex modal injection and separation at the input and the output of the field, preventing a low cost approach and furthermore, making it very difficult to control the coupling between propagation modes. Therefore, this alternative has been discarded in this paper.

The output power of a single CW laser (optical wavelength $\lambda_{D}$ ) is divided into $N$ equal parts. Each one is modulated by a different signal, which can be either baseband I-Q in the case of CPRI or radiofrequency for a RoF approach. The figure illustrates the particular case of a multiple input multiple output (MIMO) transmission link, where each signal is then injected to each one of the $N$ different cores employed for downstream transmission using subcarriers with the same RF frequency $f_{R F 1}$. At the end of the $L-\mathrm{km}$ fiber link (antenna mast) each signal is detected by a separate receiver and processed by the RRH before being radiated. The link however is versatile enough to accommodate different RF signals centered at subcarriers with different RF frequencies in each core. Note that a single core is also employed to distribute the $\mathrm{CW}$ laser (optical wavelength $\lambda_{U}$ ) for upstream transmission and, possibly, a control and supervision channel. This CW signal is evenly divided between the $N$ cores employed as a common optical carrier for upstream transmission where each of the $N$ antennas receives its particular signal and modulates the common carrier after processing it at the RRH. The independent received channels are then sent to the CO. For interfacing the MCF to the external equipment, multicore fiber connectors (MCFCs) are considered.

\section{B. Analog MCF Link design}

Each core supports, in essence, an analog radio over fiber, intermediate frequency (IF) or digital baseband link from the $\mathrm{CO}$ to the $\mathrm{BS}$ in downstream transmission and from the BS to the $\mathrm{CO}$ in upstream transmission. Ideally, for true parallel operation the cores in the MCF should be completely uncoupled. In practice, inter-core coupling must be taken into account in the design of each individual core link. For a standard analog link the performance is usually described in terms of the electrical carrier to noise ratio or CNR [8], [9]:

$$
C N R=\frac{(m \Re P)^{2} / 2}{\left\langle\Delta i_{\text {shot }}^{2}\right\rangle+\left\langle\Delta i_{\text {th }}^{2}\right\rangle+\left\langle\Delta i_{R I N}^{2}\right\rangle+\left\langle\Delta i_{\text {imd }}^{2}\right\rangle},
$$

where $m$ is the modulation index, $\mathfrak{R}$ the detector responsivity, $P$ the average optical power on the photodiode and $\left\langle\Delta i_{\text {shot }}^{2}\right\rangle$, $\left\langle\Delta i_{t h}^{2}\right\rangle,\left\langle\Delta i_{R I N}^{2}\right\rangle,\left\langle\Delta i_{i m d}^{2}\right\rangle$ the RMS powers associated to shot, thermal, relative intensity noises and the intermodulation distortion, respectively. For the pure noise sources:

$$
\begin{aligned}
& \left\langle\Delta i_{\text {shot }}^{2}\right\rangle=2 e \Re P \Delta f, \\
& \left\langle\Delta i_{\text {th }}^{2}\right\rangle=\frac{4 k_{B} T F_{n} \Delta f}{R_{L}}, \\
& \left\langle\Delta i_{R I N}^{2}\right\rangle=R I N(\Re P)^{2} \Delta f,
\end{aligned}
$$

Where $e$ is the electron charge, $k_{B}$ the Boltzman constant, $T$ the temperature, $R I N$ the relative intensity noise of the laser, $F_{n}$ and $R_{L}$ represent the noise figure and the load resistor of the receiver, respectively, and $\Delta f$ the detector bandwidth. If inter-core crosstalk is taken into account, then (1) must be modified. For instance, the link equation for core " $n$ " and low crosstalk values will be given by: 


$$
\begin{aligned}
& C N R_{n}=\frac{\left(m \Re P_{n}\right)^{2} / 2}{\left\langle\Delta i_{\text {shot }, n}^{2}\right\rangle+\left\langle\Delta i_{t h, n}^{2}\right\rangle+\left\langle\Delta i_{R I N, n}^{2}\right\rangle+\left\langle\Delta i_{\text {imd, }, n}^{2}\right\rangle+\left\langle\Delta i_{X T A L K, n}^{2}\right\rangle} \\
& =\frac{1}{C N R_{\text {shot }, n}+\frac{1}{C N R_{t h, n}}+\frac{1}{C N R_{R I N, n}}+\frac{1}{C N R_{\text {imd }, n}}+\frac{1}{C N R_{X T A L K, n}}},
\end{aligned}
$$

where $\left\langle\Delta i_{X T A L K, n}^{2}\right\rangle$ is the RMS power associated to the equivalent noise due to crosstalk from all the cores $j \neq n, P_{n}$ is the average optical power at the output of the $n$-th core and:

$$
C N R_{u, n}=\frac{\left(m \Re P_{n}\right)^{2} / 2}{\left\langle\Delta i_{u, n}^{2}\right\rangle}
$$

Represents the carrier to noise ratio due to the particular noise source $u, u=\{$ shot, th, RIN, imd, XTALK $\}$.

To compute the crosstalk contribution we need to know $\left|\Delta P_{n, k}\right|$, the value of the amplitude of the optical power fluctuations due to crosstalk from core $k$ at the output of core $n$ [10], [11]. The quotient between $\left|\Delta P_{n, k}\right|$ and $m P_{n}$ is known as the amplitude crosstalk transfer function $X T T F$ :

$$
\left|X \operatorname{TTF}_{n, k}(\omega)\right|=\left|\frac{\Delta P_{n, k}}{m P_{n}}\right| .
$$

Empirical expression for its average and variance values have been derived in [10]:

$$
\begin{aligned}
& \left\langle\left|\operatorname{XTTF}_{n, k}(\omega)\right|\right\rangle=\left\langle\mid \frac{\Delta P_{n, k}}{m P_{n}}\right\rangle=\frac{2 \kappa^{2} R L}{\beta D_{m n}} \sqrt{\left[0.31+0.69 \operatorname{sinc}^{2}\left(\frac{\omega\left(\beta_{1, n}-\beta_{1, k}\right) L}{2}\right)\right]}, \\
& \operatorname{Var}\left\{\left|X_{T T F_{n, k}}(\omega)\right|\right\}=\left(\frac{2 \kappa^{2} R L}{\beta D_{n k}}\right)^{2}\left[0.19+0.81 \operatorname{sinc}^{2}\left(\frac{\omega\left(\beta_{1, n}-\beta_{1, k}\right) L}{2}\right)\right] .
\end{aligned}
$$

In (6), $\omega$ represents the signal angular frequency, $\kappa$ is the coupling coefficient between cores $n$ and $k$ (a typical value range is 0.001 to $0.01 \mathrm{~m}^{-1}$ ), $R$ is the MCF curvature radius (a typical value ranges between 0.2 and $0.5 \mathrm{~m}), L$ is the MCF length (for typical access networks ranges between 2 and 25 $\mathrm{km}$ ), $D_{n, k}$ is the separation between cores $n$ and $k$ (a typical value ranges between 35 and $45 \mu \mathrm{m}$ ) and $\beta$ is the propagation constant of the fundamental mode in cores $n$ and $k$, which is assumed to be equal (a typical value ranges between 7-10 $\mathrm{rad} / \mu \mathrm{m})$. The parameter $d_{n, k}=\beta_{1, k}-\beta_{1, n}=1 / v_{g, n}-1 / v_{g, k}$ is the so-called skew per unit of length between cores $n$ and $k$, and depends on the group velocities in both cores (a typical value is in the range of 0.01 and $0.1 \mathrm{ps} / \mathrm{km})$.

Now, since $\left|\Delta i_{n, k}\right|=\mathfrak{R}\left|\Delta P_{n, k}\right|$ it follows that $\Delta i_{n, k}^{2}=$ $\mathfrak{R}^{2}\left|\Delta P_{n, k}\right|^{2}$ and therefore:

$$
\frac{1}{C N R_{X T A L K, n, k}}=\frac{2\left\langle\Delta i_{n, k}^{2}\right\rangle}{\left(m \Re P_{n}\right)^{2}}=\frac{2\left\langle\left|\Delta P_{n, k}\right|^{2}\right\rangle}{\left(m P_{n}\right)^{2}}=2\left\langle\left|X T T F_{n, k}(\omega)\right|^{2}\right\rangle .
$$

The total crosstalk in core $n$ is due to the contribution of the rest of the cores in the MCF:

$$
\frac{1}{C N R_{X T A L K, n}}=\sum_{k \neq n} \frac{1}{C N R_{X T A L K, n, k}}=2 \sum_{k \neq n}\left\langle\left|X T T F_{n, k}(\omega)\right|^{2}\right\rangle=\sum_{k \neq n} H_{n, k} .
$$

To compute $H_{n, k}$, we make use of (6):

$$
\begin{gathered}
H_{n, k}=2\left\langle\left|X \operatorname{XTF}_{n, k}(\omega)\right|^{2}\right\rangle=2\left\{\operatorname{Var}\left\{\left|X T T F_{n, k}(\omega)\right|\right\}+\left[\left\langle X T T F_{n, k}(\omega) \mid\right\rangle\right]^{2}\right\}= \\
=\left(\frac{2 \kappa^{2} R L}{\beta D_{n k}}\right)^{2}\left[1+3 \operatorname{sinc}^{2}\left(\frac{\omega\left(\beta_{1, n}-\beta_{1, k}\right) L}{2}\right)\right] .
\end{gathered}
$$

For the evaluation of (8) it is customary to take an upper bound corresponding to the worst case [7], [11], [12], which in typical hexagonal 7- and 19-core MCFs corresponds to the central core $(n=1)$. For the case of a 7 -core MCF, the distance $D$ between the central and surrounding cores is the same, hence, if we assume an equal skew per unit of length between the central and the surrounding cores we have $H_{1, k}=$ $H_{1}, \forall k$. Since only half of the cores contribute to upstream/downstream transmission then:

$$
\left.\frac{1}{C N R_{X T A L K}}\right|_{7 C-M C F}=3 H_{1} .
$$

For the case of a 19-core MCF, if the 6 internal surrounding cores are at a distance $D$ from the central core, there are other 6 at a distance $2 D$ and another 6 at a distance $\sqrt{3} D$. Thus we have:

$$
\left.\frac{1}{C N R_{X T A L K}}\right|_{19 C-M C F}=\frac{19 H_{1}}{4} .
$$

Introducing (10) or (11) in (3) renders the complete CNR expression that takes into account the impact of the inter-core crosstalk.

Fig. 2 shows the value of $1 / C N R_{X T A L K}$ as a function of the modulating frequency taking the link length $L$ as a parameter, for a standard 19-core MCF with $\kappa=0.01 \mathrm{~m}^{-1}, R=0.5 \mathrm{~m}, D_{n, k}$ $=44 \mu \mathrm{m}, \beta=6 \mathrm{rad} / \mu \mathrm{m}$ and $d_{n, k}=0.03 \mathrm{ps} / \mathrm{km}$.

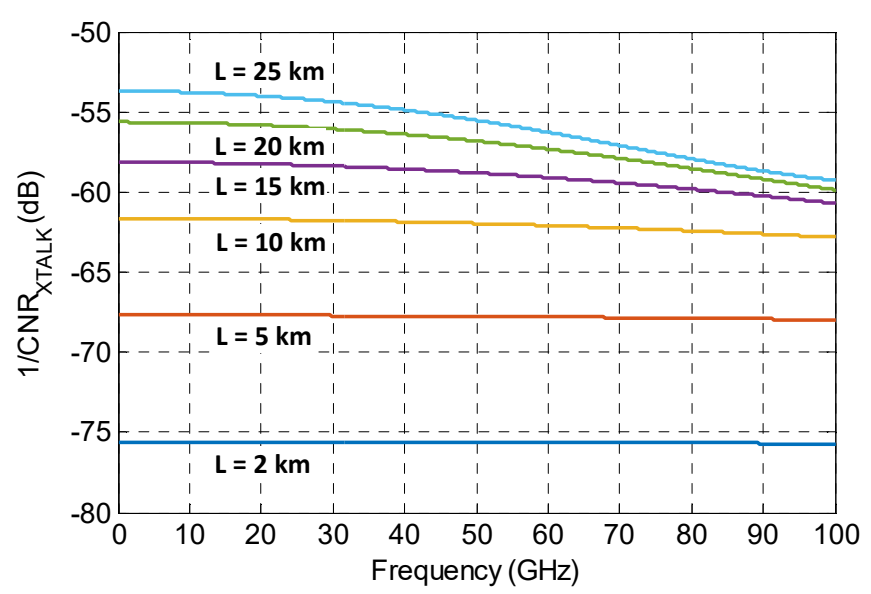

Fig. 2. $1 / C N R_{X T A L K}$ as a function of the modulating frequency taking the link length $L$ as a parameter, for a standard 19-core MCF with $\kappa=0.01 \mathrm{~m}^{-1}, R=0.5$ $\mathrm{m}, D_{n, k}=44 \mu \mathrm{m}, \beta=6 \mathrm{rad} / \mu \mathrm{m}$ and $d_{n, k}=0.03 \mathrm{ps} / \mathrm{km}$. 
As it can be observed, relevant electrical crosstalk levels (comparable to other noise sources if $>-65 \mathrm{~dB}$ [MWP]) can be achieved for moderate link distances $(>10 \mathrm{~km})$. For short RAN links, below $10 \mathrm{~km}$, the impact of crosstalk can be safely considered as negligible as compared to other noise sources.

However, these levels can be reduced either by reducing the values of the coupling coefficients between cores or by reducing the value of the critical bending radius. To achieve this, trench-type MCFs have been proposed and demonstrated [11]. Fig. 3 shows the value of $1 / C N R_{X T A L K}$ as a function of frequency taking the link length $L$ as a parameter, for the same case as in Fig. 2 when the coupling coefficient is reduced from $\kappa=0.01 \mathrm{~m}^{-1}$ to $\kappa=0.002 \mathrm{~m}^{-1}$ (upper) and when the critical bending radius is reduced from $R=0.5$ to $0.2 \mathrm{~m}$ (lower).
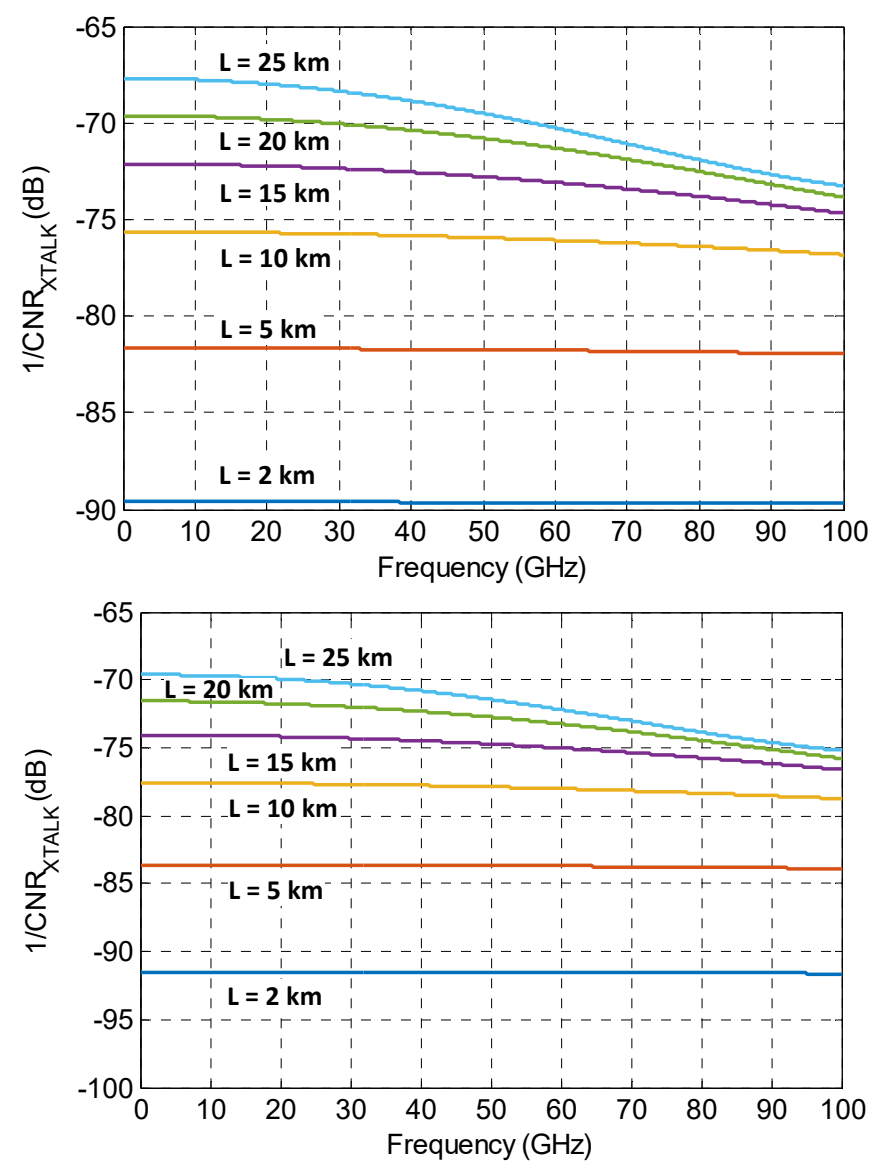

Fig. 3. $1 / C N R_{X T A L K}$ as a function of frequency taking the link length $L$ as a parameter, for a standard 19-core MCF. Upper: $\kappa=0.002 \mathrm{~m}^{-1}, R=0.5 \mathrm{~m}, D_{n, k}$ $=44 \mu \mathrm{m}, \beta=6 \mathrm{rad} / \mu \mathrm{m}$ and $d_{n, k}=0.03 \mathrm{ps} / \mathrm{km}$. Lower: $\kappa=0.01 \mathrm{~m}^{-1}, R=0.2 \mathrm{~m}$, $D_{n, k}=44 \mu \mathrm{m}, \beta=6 \mathrm{rad} / \mu \mathrm{m}$ and $d_{n, k}=0.03 \mathrm{ps} / \mathrm{km}$.

In both cases and even for link lengths over $10 \mathrm{~km}$ the crosstalk levels are below $<-70 \mathrm{~dB}$, which in practice is a negligible value. For links above $10 \mathrm{~km}$, the crosstalk performance must be considered but its behavior can be quite frequency dependent as recently reported in [10], this is a consequence of the increase of the skew $d_{n, k} L$ between coupled cores. This feature is slightly apparent and also depends on the value of the skew per unit length value, which can range from 0.01 to $0.1 \mathrm{ps} / \mathrm{km}$.

Fig. 4 shows the crosstalk as a function of the signal frequency for a standard 19-core MCF with $\kappa=0.01 \mathrm{~m}^{-1}, R=$ $0.5 \mathrm{~m}, D_{n, k}=44 \mu \mathrm{m}$ and $\beta=6 \mathrm{rad} / \mu \mathrm{m}$. The upper figure corresponds to a link length of $L=10 \mathrm{~km}$, while the lower part is for $L=25 \mathrm{~km}$.

The high-pass filtering effect is clear in both figures and can be explained because as frequency increases there is an increase in the differential phase between signals travelling through the coupling cores, leading to phase mismatch.

The equations developed in this section correspond to a worst case scenario as we have assumed MCF characteristics associated with spooled fibers whereas in a real network, the fibers would be unspooled. Although the true characteristics of unspooled fibers are yet to be known, one may assume that spooled fibers correspond to an upper bound in the crosstalk value.

The main conclusion of this section is that inter-core crosstalk noise will only be significant if the link length is $>10$ $\mathrm{km}$ and if a non-optimized MCF design is employed. In practice, since around a $96 \%$ of the access network links are below $10 \mathrm{~km}$, this means that the impact of crosstalk can be neglected in most cases and link design can be carried independently core by core.
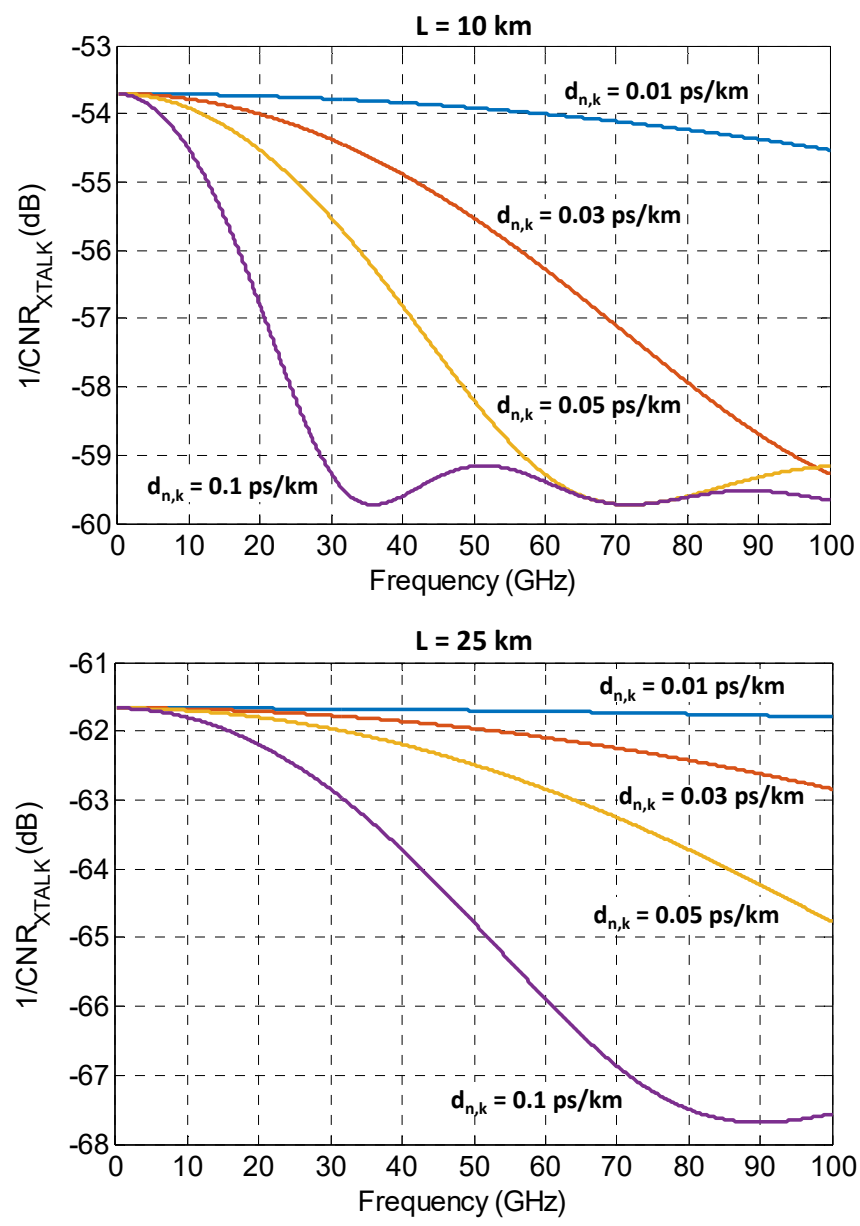

Fig. 4. $1 / C N R_{X T A L K}$ as a function of frequency taking the skew per unit length value $d_{n, k}$ as a parameter, for a standard 19-core MCF with $\kappa=0.01 \mathrm{~m}^{-1}, R=$ 
$0.5 \mathrm{~m}, D_{n, k}=44 \mu \mathrm{m}, \beta=6 \mathrm{rad} / \mu \mathrm{m}$ and $d_{n, k}=0.03 \mathrm{ps} / \mathrm{km}$. Upper: $L=10 \mathrm{~km}$, Lower: $L=25 \mathrm{~km}$.

\section{SDM-BASED C-RAN FRONT-HAUL ARCHITECTURES}

\section{A. Radio over Fiber operation}

The single CO-BS SDM-based link described in the previous section can serve as the basic sector connection for RoF access networks. Fig. 5 shows a representative C-RAN distribution architecture where the $\mathrm{CO}$ is connected to each one of the sectors conforming the different BSs by means of $2 N+1$-core MCFs, $(N$ cores for downstream transmission and $N$ cores for upstream transmission). The $i$-th input core to/from sector $j$ in $\mathrm{BS}_{k}$ is labeled as $s_{i}^{k, j}$, as shown in the expanded picture of a MCF link displayed in the lower part of Fig. 5. A centralized switch placed in the $\mathrm{CO}$ provides the dynamic mapping of/to downstream/upstream subcarrier channels and bands to/from the spatial ports. The fabric includes an internal electronic core where SDN switching is performed prior to the modulation of the $\lambda_{D}$ laser in provides capacity expansion by using $N \mathrm{x} N$ MIMO over only one frequency allocated in band $1\left(f_{R F}^{1,1}\right) ; \mathrm{BS}_{2}$ provides capacity expansion by carrier aggregation using three carriers in band $1\left(f_{R F}{ }^{1,1}, f_{R F}{ }^{1,2}, f_{R F}{ }^{1, S_{1}}\right)$ in the three sectors and only one radiator per sector (no MIMO); while $\mathrm{BS}_{3}$ features capacity expansion by using $N \mathrm{x} N$ MIMO in band $1\left(f_{R F}^{1, \mathrm{~S}_{1}}\right)$, carrier aggregation in band $2\left(f_{R F}^{2,2}, f_{R F}{ }^{2, \mathrm{~S}_{2}}\right)$ and $2 \times 2$ MIMO in band $M$ $\left(f_{R F}{ }^{M, 2}\right)$.

\section{B. Digitized Radio over Fiber operation}

Fig. 6 shows the proposed configuration of a C-RAN architecture based on the CO-BS basic link applied to DRoF operation. In this case, the input (downstream signal)/output (upstream signal) to/from the electronic switch placed at the $\mathrm{CO}$ corresponds to a BBU pool where a series of virtual BBUs are defined by software to service $\mathrm{BS}_{1}$ to $\mathrm{BS}_{M}$. Each virtual BBUm, with $m=1$ to $M$, can be reconfigured to allocate different capacities dynamically. Resources are allocated to the virtual BBUs by an overall manager, and each virtual BBU is directly tied to specific cores of a given MCF.
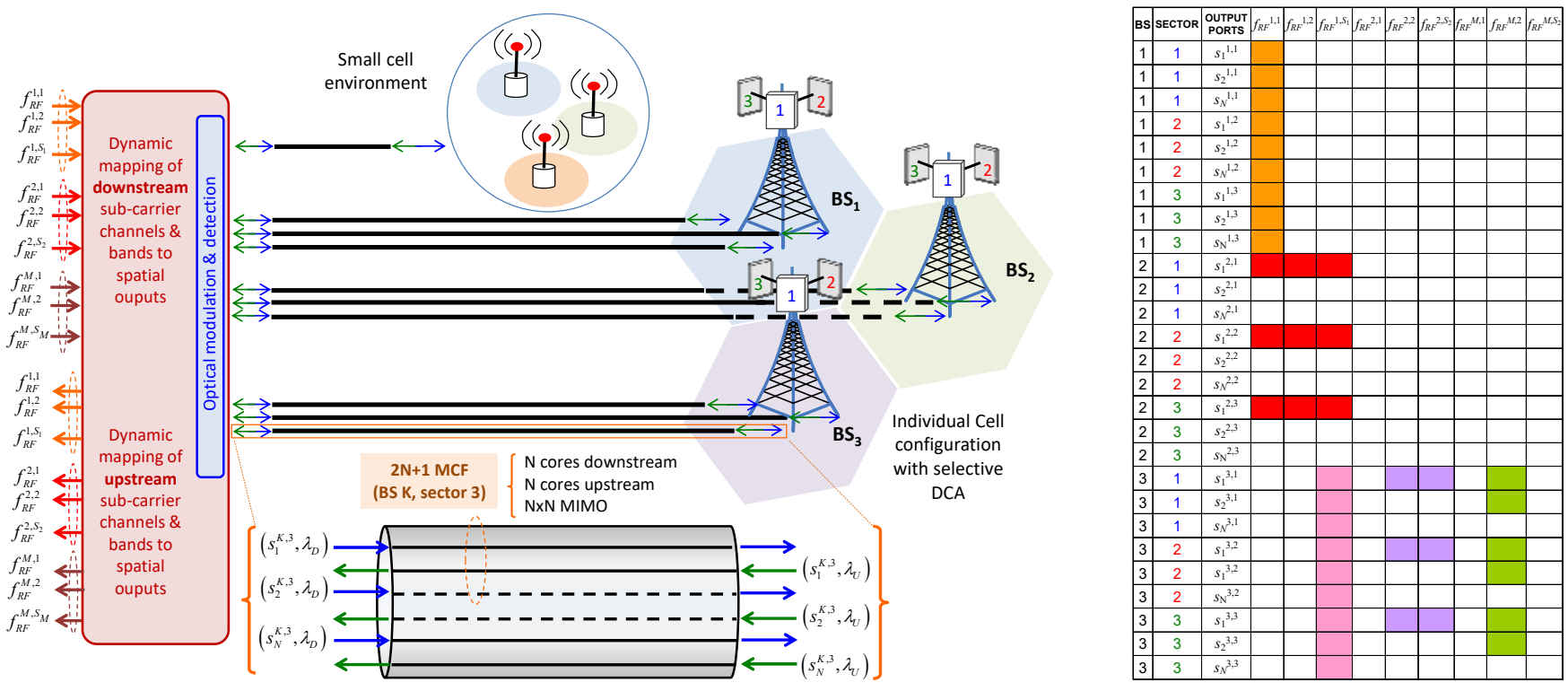

Resource allocation table example

Fig. 5. (Left) MCF-based C-RAN configuration for RoF operation. (Right) Example of a resource allocation table.

downstream and after detection of the $\lambda_{U}$ signal in upstream.

We must note that the spatial diversity available at the $\mathrm{CO}$ output provides several advantages. First, each sector within a given BS can be independently addressed and softwaredefined configured with a MCF having a similar outer diameter as a SMF-28, thus avoiding fiber bundling. Second, resource allocation can be implemented electronically enabling both carrier aggregation and MIMO operation. Third, the number of MIMO radiators in a given sector can be independently and dynamically set from 1 to $N$ at the CO, enabling, for instance, CoMP from adjacent sectors in neighboring BSs.

As an example, the resource allocation table on Fig. 5 shows a representative switching configuration where $\mathrm{BS}_{1}$
The resource allocation table illustrated in Fig. 6 shows two representative examples of resource reconfigurations given in a DRoF scenario. In both cases we assume equal capacity/sector at each BS. In the upper table we illustrate the case of carrier aggregation over multi-band non-contiguous (800-900 MHz) $10+10 \mathrm{MHz}$ for LTE-A using different configurations of MIMO. If only one antenna is active, the CPRI bit rate is $1.536 \mathrm{~Gb} / \mathrm{s}$ that is actually illustrated as a basic CPU unit. For instance, $\mathrm{BS}_{1}$ and $\mathrm{BS}_{2}$ implement independent $4 \times 4$ and $2 \times 2$ MIMO with an overall capacity per sector of 6.14 and $3.07 \mathrm{~Gb} / \mathrm{s}$, respectively. On the other hand, the set conformed by $\mathrm{BS}_{M-2}, \mathrm{BS}_{M-1}$ and $\mathrm{BS}_{M}$ implements a supercell with $8 \times 8$ MIMO and an overall capacity per sector of $12.28 \mathrm{~Gb} / \mathrm{s}$. The cumulative capacity employed in terms of 
$\%$ of CPU usage of the BBU pool is illustrated in grey.

The lower table illustrates a situation where the capacity is being reassigned. Here single-band non-contiguous (900 negligible) spectral behavior regarding crosstalk should be expected in most practical situations in the spectral region spanning from baseband to $10 \mathrm{GHz}$, therefore this

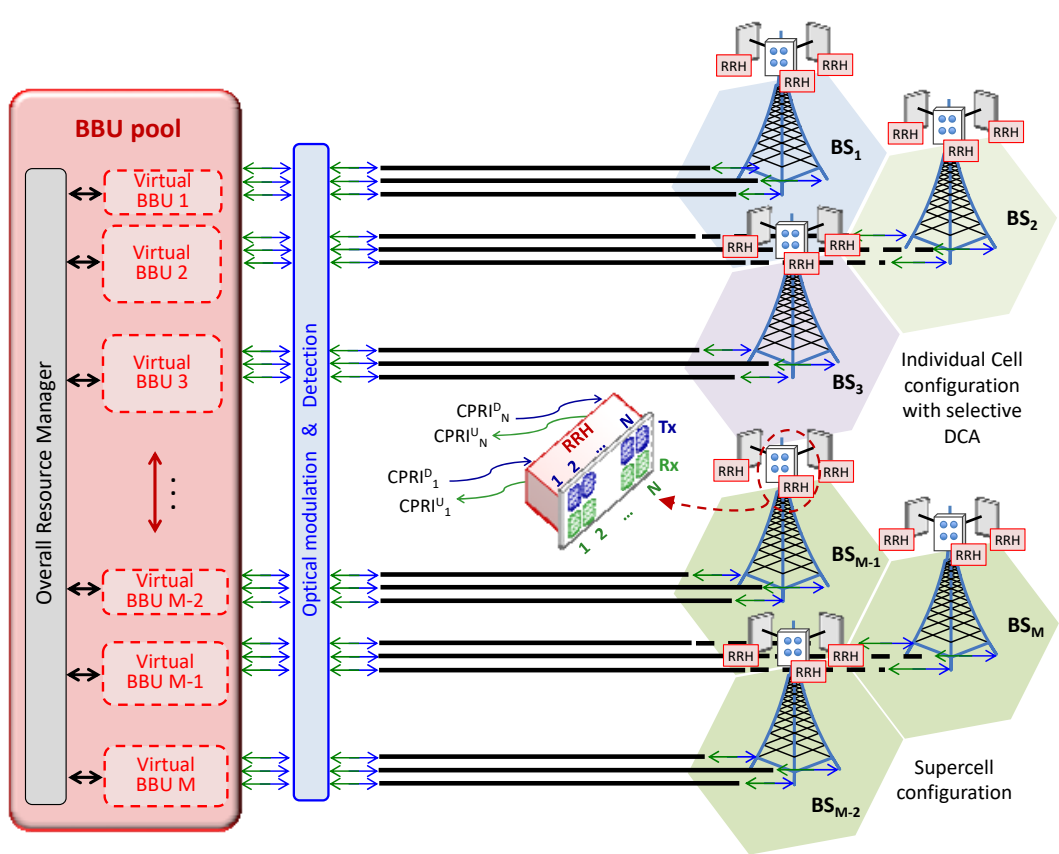

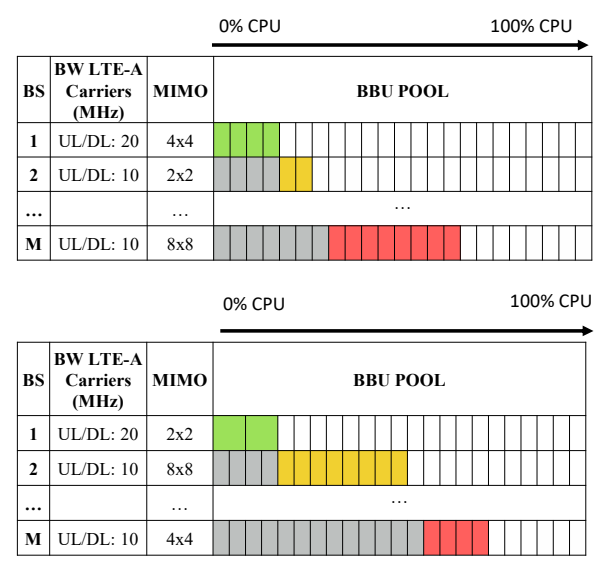

Resource allocation table examples

Fig. 6. (Left) MCF-based C-RAN configuration for DRoF operation. (Right) Example of a resource allocation table.

$\mathrm{MHz}) 5+5 \mathrm{MHz}$ for LTE-A is supported using different configurations of MIMO. If only one antenna is active, the CPRI bit-rate is $0.768 \mathrm{~Gb} / \mathrm{s}$ that is illustrated as a basic unit.

Bit rates are kept to $1.536 \mathrm{~Gb} / \mathrm{s}$ for the cores feeding $\mathrm{BS}_{1}$ and its overall capacity per sector is now $3.07 \mathrm{~Gb} / \mathrm{s}$ while the number of radiators is changed from 4 to 2 ; for $\mathrm{BS}_{2}$ the number of radiators changes from 1 to 8 , the bit rate per core is $0.768 \mathrm{~Gb} / \mathrm{s}$ while the overall capacity per sector is increased to $6.14 \mathrm{~Gb} / \mathrm{s}$. Finally, the supercell implemented by $\mathrm{BS}_{M-2}$, $\mathrm{BS}_{M-1}$ and $\mathrm{BS}_{M}$ provides a capacity of $3.07 \mathrm{~Gb} / \mathrm{s}$ per sector.

\section{PON INTEGRATION}

The proposed MCF-based RAN architecture brings an additional advantage since due to the spatial diversity provided by the MCFs it can easily integrate a passive optical network architecture. The most straightforward configuration is that shown in Fig. 7.

Here, using $2 N-2$ cores for the downstream/upstream MIMO transmission, and 1 core for the upstream $\mathrm{CW}$ laser distribution, leaves two free cores to support the downstream and upstream transmission required by a PON. Each one of these can carry digital baseband information using the regulated wavelength pertaining to each propagation direction. In the configuration displayed in Fig. 7 the downstrean/upstream cores are connected to $1 \mathrm{xM}$ couplers for further power division/sum respectively, but they cold be directly tied to a final optical network unit (ONU).

The results obtained in Section II indicate that uniform (and configuration should be, in principle capable of supporting

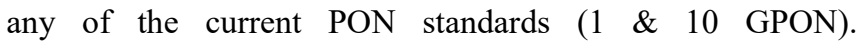
Furthermore, as the transmission in each core is compatible with a WDM overlay, it will also be capable of supporting emerging WPON standards.

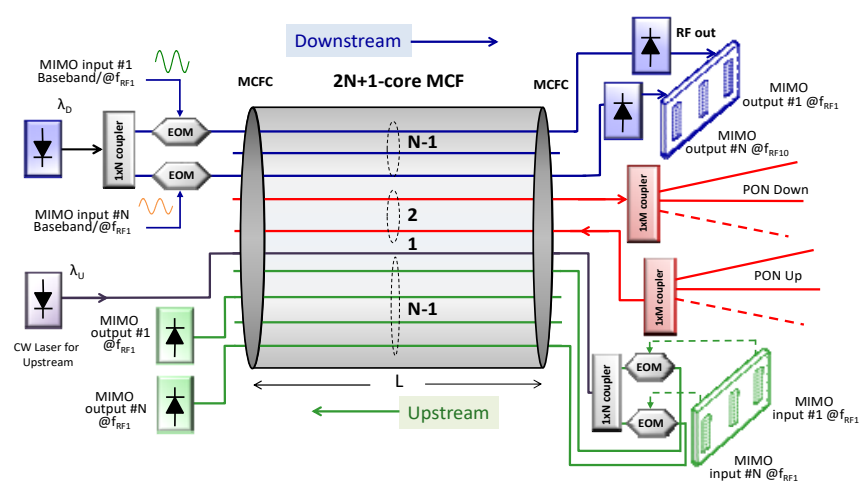

Fig. 7. MCF-based C-RAN configuration incorporating PON integration.

As an example, Table I shows possible configurations where C-RAN with MIMO, control channels and PON are directly integrated for different MCF models. For each case the maximum MIMO diversity is displayed in the second column.

TABLE I

MIMO C-RAN AND PON INTEGRATION OPTIONS FOR DIFFERENT MCF DESIGNS 


\begin{tabular}{cccc}
\hline \hline MCF Design & MIMO & PON & Control \\
\hline 7-Core Homogeneous & $2 \times 2$ & 2 & 1 \\
12-Core Homogeneous & $3 \times 3$ & 2 & 1 \\
19-Core Homogeneous & $8 \times 8$ & 2 & 1 \\
36-Core Homogeneous & $16 \times 16$ & 2 & 1 \\
\hline \hline
\end{tabular}

\section{CONCLusions}

We have proposed MFCs to support C-RAN architectures capable of addressing their main present and future challenges through the exploitation of spatial division multiplexing. This novel approach allows SDN and NFV and is compatible with both WDM and PON overlay expansion. SDN and NFV implementation can be achieved by exploiting the fact that both in the DRoF and RoF approaches the traffic and capacity characteristics of each sector in each BS can be reconfigured by software in the electrical domain through an overall resources manager located in the electronic switch (shown in figure 6). Furthermore, the proposed architecture is potentially integrable with MCF-based solutions for metro networks if architecture on demand (AoD) gateway nodes [13] are introduced at the $\mathrm{CO}$. A possible solution would require spatial switching and could be built upon the process described in [14].

\section{ACKNOWLEDGMENT}

We thank the research funding supported by GVA PROMETEO 2013/012 and TEC2014-60378-C2-1-R projects.

\section{REFERENCES}

[1] China Mobile Research Institute, "C-RAN: The road towards green ran", available at http://labs.chinamobile.com.

[2] P. Chanclou et al., "Optical Fiber Solution for Mobile Fronthaul to Achieve Cloud Radio Access Network," in Proc. Future Network \& Mobile Summit Conf. 2013, Lisbon, Portugal, 2013.

[3] A. Pizzinat et al., "Things you should know about Fronthaul," $J$. Lightwave Technol., vol. 33, pp. 1077-1083, 2015.

[4] A. Saadani et al., "Digital Radio over Fiber for LTE-Advanced: Opportunities and Challenges," in Proc. ONDM 2013, Brest, France, 2013, pp. 194.

[5] J. E. Mitchell, "Integrated wireless backhaul over optical access networks," IEEE J. Lightwave Technol., vol. 32, pp. 3373-3382, 2014.

[6] C. Liu et al., "Key Microwave-Photonics Technologies for NextGeneration Cloud-Based Radio Access Networks," IEEE J. Lightwave Technol., vol. 32, pp. 3452-3460, 2014.

[7] M. Koshiba, "Design aspects of multicore optical fibers for highcapacity Long-Haul transmission," in Proc. IEEE Top. Meeting MWP 14, Sapporo, Japan, 2014, pp. 318.

[8] J. Capmany and D. Novak, "Microwave photonics combines two worlds," Nat. Photonics, vol. 1, no. 6, pp. 319-330, 2007.

[9] J. Yao, "Microwave photonics," IEEE J. Lightwave Technol., vol. 27, no. 3, pp. 314-335, 2009.

[10] R.S. Luis, B.J. Puttnam, A. V. Cartaxo, W. Klaus, J.M. Mendinueta, Y. Awaji, N. Wada, T. NNakanishi, T. Hayashi and T. Sasasaki, "Time and modulation frequency dependence of crosstalk in homogeneous multicore fibers," IEEE J. Lightwave Technol., in press.

[11] T. Hayashi, T. Taru, O. Shimakawa, T. Sasaki, and E. Sasaoka, "Design and fabrication of ultra-low crosstalk and low-loss multi-core fiber," Opt. Express, vol. 19, pp. 16576-16592, 2011.

[12] M. Koshiba, K. Saitoh, K. Takenaga, and S. Matsuo, "Multi-core fiber design and analysis: coupled-mode theory and coupled-power theory," Opt. Express, vol. 19, no. 26, p. B102, Dec. 2011
[13] N. Amaya et al., "Software defined networking over space division multiplexing optical networks: features, benefits and experimental demonstration," Opt. Express, vol. 22, pp. 3638-3647, 2014.

[14] N. Amaya, et al., "Fully-elastic multi-granular network with space/frequency/time switching using multi-core fibres and programmable optical nodes," Opt. Express, vol. 21, pp. 8865-8872, 2013. 